\title{
Highly entangled quantum spin chains and their semigroup extensions
}

\author{
Fumihiko Sugino* \\ Center for Theoretical Physics of the Universe, Institute for Basic Science \\ Expo-ro 55, Yuseong-gu, Daejeon 34126, Republic of Korea \\ E-mail: fusugino@gmail.com

\section{Pramod Padmanabhan} \\ Center for Theoretical Physics of Complex Systems, Institute for Basic Science \\ Expo-ro 55, Yuseong-gu, Daejeon 34126, Republic of Korea \\ E-mail: pramod23physegmail.com
}

\section{Vladimir Korepin}

C.N. Yang Institute for Theoretical Physics, Stony Brook University

NY 11794, USA

E-mail: korepin@gmail.com

\begin{abstract}
We discuss spin chain models exhibiting extraordinary large entanglement which violates the area law by a square root of the volume. It is striking because the violation had been believed to be at most logarithmic over twenty years. Next, we construct extended models of the spin chains based on symmetric inverse semigroups. It will be interesting to apply these models to problems in quantum gravity or black holes.
\end{abstract}

The 39th International Conference on High Energy Physics (ICHEP2018)

4-11 July, 2018

Seoul, Korea

${ }^{*}$ Speaker. 


\section{Introduction}

Quantum entanglement appears ubiquitously in quantum mechanics, and gives a correlation which cannot be explained in classical mechanics. The superposition principle of states in quantum mechanics is a key to the entanglement. One of famous examples of entangled states is the EinsteinPodolsky-Rosen (EPR) state that is a state of a system of two particles $A$ and $B$ taking spins $\uparrow$ and $\downarrow:|\psi\rangle=\left(|\uparrow\rangle_{A}|\downarrow\rangle_{B}+|\downarrow\rangle_{A}|\uparrow\rangle_{B}\right) / \sqrt{2}$. We cannot say definitely about the spins of $A$ or $B$ before doing measurement. At the moment that the spin of $A$ is measured, the spin of $B$ will be uniquely determined in spite that the spin of $B$ is not directly measured. This happens instantaneously even though $A$ and $B$ are far away from each other. It is due to that the state $|\psi\rangle$ cannot be expressed as a direct product of a state of $A$ and a state of $B$. Similarly in quantum many body system on a lattice or quantum field theory, by dividing a space that the theory defined on into two regions (called $A$ and $B$ ), we can consider the entanglement between them.

Entanglement entropy (EE) is a measure of the entanglement, i.e. quantifying how far the state of a total system is from a tensor product of states of subsystems. The reduced density matrix of the subsystem $A$ is obtained by tracing out the states of $B$ in the density matrix of the total system $\rho: \rho_{A}=\operatorname{Tr}_{B} \rho$. Then, the EE of $A$ is defined as the von Neumann entropy of $\rho_{A}: S_{A}=\operatorname{Tr}_{A}\left(\rho_{A} \ln \rho_{A}\right)$. For the EPR state, $\rho=|\psi\rangle\langle\psi|, \rho_{A}=(|\uparrow\rangle\langle\uparrow|+| \downarrow\rangle\langle\downarrow|) / 2, S_{A}=\ln 2$.

Let us consider the ground state of a quantum many-body system with local interactions in $D$ spatial dimensions. The $\mathrm{EE}$ of the ground state is typically proportional to the area of the boundary between $A$ and $B$ (area law), scaling as $O\left(L^{D-1}\right)$ with $L$ being a length scale of $A$ [1]. This is understandable from the fact that local interactions contributing to the EE localize around the boundary for gapped systems ${ }^{1}$. On the other hand, violations of the area law are observed in gapless systems, whose amount had been believed to be a logarithm, i.e. scaling as $O\left(L^{D-1} \ln L\right)$, over twenty years. Recently, Movassagh and Shor discovered a solvable one-dimensional spin system which provides the EE scaling as $O(\sqrt{L})$ and gives a counterexample of the belief [3]. In this article, we mainly discuss the model (Motzkin spin chain) and its extension by symmetric inverse semigroups (SISs) [4].

\section{Motzkin spin chain}

The Motzkin spin chain [3] of length $2 n$ has up, down and zero quantum spin degrees of freedom with multiplicity (called as color) $s$ for up and down at each of the lattice sites $\{1,2, \cdots, 2 n\}$. We express the up and down spins with the color $k \in\{1,2, \cdots, s\}$ and the zero spin at the site $i$ as $\left|u_{i}^{k}\right\rangle,\left|d_{i}^{k}\right\rangle$ and $\left|0_{i}\right\rangle$, respectively. The Hamiltonian is given by the sum of local projection operators:

$$
\begin{aligned}
H_{\mathrm{M}, s}= & \sum_{j=1}^{2 n-1} \sum_{k=1}^{s}\left\{\left|D_{j, j+1}^{k}\right\rangle\left\langle D_{j, j+1}^{k}|+| U_{j, j+1}^{k}\right\rangle\left\langle U_{j, j+1}^{k}|+| F_{j, j+1}^{k}\right\rangle\left\langle F_{j, j+1}^{k}\right|\right\} \\
& +\sum_{j=1}^{2 n-1} \Pi_{j, j+1}^{\text {cross }}+\sum_{k=1}^{s}\left(\left|d_{1}^{k}\right\rangle\left\langle d_{1}^{k}|+| u_{2 n}^{k}\right\rangle\left\langle u_{2 n}^{k}\right|\right)
\end{aligned}
$$

where

\footnotetext{
${ }^{1}$ There is a mathematical proof of the area law for gapped one-dimensional systems [2].
} 

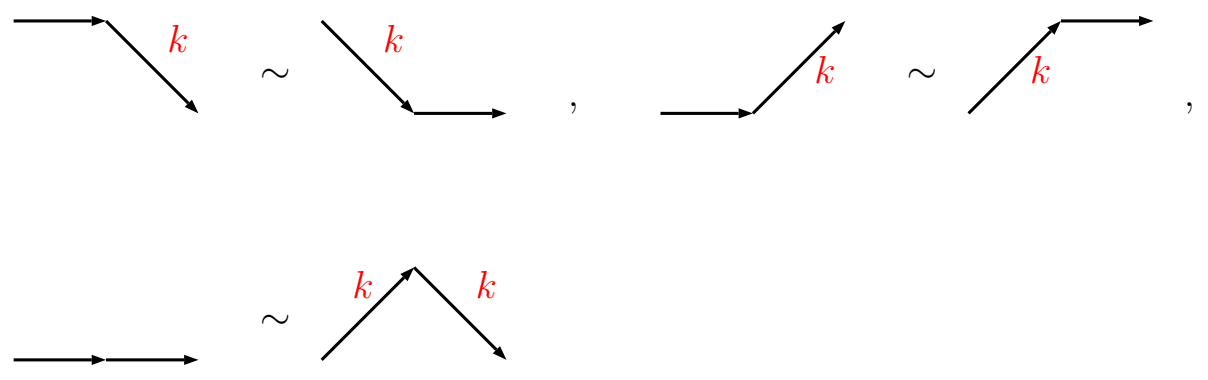

Figure 1: The walks corresponding to the ground state should be invariant under applying the three moves to arbitrary nearest neighbors.

$$
\begin{array}{rlrl}
\left|D_{j, j+1}^{k}\right\rangle & =\frac{1}{\sqrt{2}}\left(\left|0_{j}, d_{j+1}^{k}\right\rangle-\left|d_{j}^{k}, 0_{j+1}\right\rangle\right), & \left|U_{j, j+1}^{k}\right\rangle=\frac{1}{\sqrt{2}}\left(\left|0_{j}, u_{j+1}^{k}\right\rangle-\left|u_{j}^{k}, 0_{j+1}\right\rangle\right), \\
\left|F_{j, j+1}^{k}\right\rangle & =\frac{1}{\sqrt{2}}\left(\left|0_{j}, 0_{j+1}\right\rangle-\left|u_{j}^{k}, d_{j+1}^{k}\right\rangle\right), \quad \Pi_{j, j+1}^{\text {cross }}=\sum_{k \neq k^{\prime}}\left|u_{j}^{k}, d_{j+1}^{k^{\prime}}\right\rangle\left\langle u_{j}^{k}, d_{j+1}^{k^{\prime}}\right| .
\end{array}
$$

In (2.1), all the interactions are over the nearest neighbor sites as the suffix $j, j+1$ means, and the last term is the boundary term. Since each projection operator is positive semi-definite, the Hamiltonian (2.1) has a positive semi-definite spectrum. If we find a zero-energy state, it must be a ground state, which is annihilated by each of the local projectors. This property is called as frustration free. By representing the up, down and zero spin states as arrows pointing to $(1,1)$ (up-step), $(1,-1)$ (down-step) and $(1,0)$ (flat-step) respectively, each spin configuration is mapped to a walk in the $(x, y)$-plane starting at the origin and ending at $(2 n, 0)$. The up- and down-steps have the color indices. Let us represent the zero-energy ground state in terms of the walk. From the boundary term, down (up) walks are forbidden at the left (right) edge. Since the ground state is orthogonal to $\left|D_{j, j+1}^{k}\right\rangle,\left|U_{j, j+1}^{k}\right\rangle$ and $\left|F_{j, j+1}^{k}\right\rangle$, the walks corresponding to the ground state should be invariant under the three moves acting arbitrary nearest neighbors depicted in Fig. 1. The term $\Pi_{j, j+1}^{\text {cross }}$ implies that the color of each up-step should be matched with the color of a subsequent down-step appearing at the same height. Therefore, the zero-energy ground state turns out to be unique and corresponding to random walks starting at the origin, ending at $(2 n, 0)$ and not entering the $y<0$ region. Such random walks are called (colored) Motzkin walks. For example, the ground state in the $2 n=4$ case reads

$$
\begin{gathered}
\left|P_{\mathrm{M}, 4, s}\right\rangle=\frac{1}{\sqrt{1+6 s+2 s^{2}}}\left[\left|0_{1}, 0_{2}, 0_{3}, 0_{4}\right\rangle+\sum_{k=1}^{s}\left\{\left|u_{1}^{k}, d_{2}^{k}, 0_{3}, 0_{4}\right\rangle+\left|0_{1}, u_{2}^{k}, d_{3}^{k}, 0_{4}\right\rangle+\left|0_{1}, 0_{2}, u_{3}^{k}, d_{4}^{k}\right\rangle\right.\right. \\
\left.+\left|u_{1}^{k}, 0_{2}, d_{3}^{k}, 0_{4}\right\rangle+\left|0_{1}, u_{2}^{k}, 0_{3}, d_{4}^{k}\right\rangle+\left|u_{1}^{k}, 0_{2}, 0_{3}, d_{4}^{k}\right\rangle\right\} \\
\left.+\sum_{k, \ell=1}^{s}\left\{\left|u_{1}^{k}, d_{2}^{k}, u_{3}^{\ell}, d_{4}^{\ell}\right\rangle+\left|u_{1}^{k}, u_{2}^{\ell}, d_{3}^{\ell}, d_{4}^{k}\right\rangle\right\}\right] .
\end{gathered}
$$

The number $1+6 s+2 s^{2}$ appearing in the normalization factor gives the number of the colored Motzkin walks. In general, the number of the length- $2 n$ colored Motzkion walks is given by

$$
N_{\mathrm{M}, 2 n, s}=\sum_{\rho=0}^{n}\left(\begin{array}{c}
2 n \\
2 \rho
\end{array}\right) s^{n-\rho} \frac{1}{n+1}\left(\begin{array}{c}
2 n-2 \rho \\
n-\rho
\end{array}\right) .
$$


We take the left half of the system as a subsystem $A(A=\{1,2, \cdots, n\})$ and the remaining as $B$ $(B=\{n+1, n+2, \cdots, 2 n\})$. In order to compute the EE of $A$, we rewrite the ground state $\left|P_{\mathrm{M}, 2 n, s}\right\rangle$ as sums of direct products of a state belonging to $A$ and a state belonging to $B$ :

$$
\left|P_{\mathrm{M}, 2 n, s}\right\rangle=\sum_{h=0}^{n} \sum_{\kappa_{1}=1}^{s} \cdots \sum_{\kappa_{h}=1}^{s} \sqrt{p_{\mathrm{M}, n, n, s}^{(h)}}\left|\tilde{P}_{\mathrm{M}, n, s}^{(0 \rightarrow h)}(\{\kappa\})\right\rangle \otimes\left|\tilde{P}_{\mathrm{M}, n, s}^{(h \rightarrow 0)}(\{\kappa\})\right\rangle,
$$

where the state $\left|\tilde{P}_{\mathrm{M}, n, s}^{(0 \rightarrow h)}(\{\kappa\})\right\rangle$ belongs to the subsystem $A$ and corresponds to a part of the Motzkin walks starting at the origin and ending at $(n, h)$. Since the part has $h$ unmatched up-steps, $\kappa_{m}$ denotes the color of the up-step from the height $m-1$ to $m$. The state $\left|\tilde{P}_{\mathrm{M}, n, s}^{(h \rightarrow 0)}(\{\kappa\})\right\rangle$ corresponds to the remaining part of the Motzkin walks from $(n, h)$ to $(2 n, 0)$ with $h$ unmatched down-steps. Let $\tilde{N}_{\mathrm{M}, n, s}^{(0 \rightarrow h)}$ be the number of the paths in $\left|\tilde{P}_{\mathrm{M}, n, s}^{(0 \rightarrow h)}(\{\kappa\})\right\rangle$. The coefficient $p_{\mathrm{M}, n, n, s}^{(h)}$ is given by $\left(\tilde{N}_{\mathrm{M}, n, s}^{(0 \rightarrow h)}\right)^{2} / N_{\mathrm{M}, 2 n, s}$, and can be seen to have the asymptotic behavior

$$
p_{\mathrm{M}, n, n, s}^{(h)} \sim \frac{\sqrt{2} s^{-h}}{\sqrt{\pi}(\sigma n)^{3 / 2}}(h+1)^{2} e^{-\frac{1}{2 \sigma}(h+1)^{2}} \times\left[1+O\left(n^{-1}\right)\right]
$$

with $\sigma \equiv \sqrt{s} /(2 \sqrt{s}+1)$ as $n \rightarrow \infty$. For the density matrix of the ground state $\rho=\left|P_{\mathrm{M}, 2 n, s}\right\rangle\left\langle P_{\mathrm{M}, 2 n, s}\right|$, the EE of $A$ is expressed as

$$
S_{A}=\sum_{h=0}^{n} s^{h} p_{\mathrm{M}, n, n, s}^{(h)} \ln p_{\mathrm{M}, n, n, s}^{(h)} .
$$

The factor $s^{h}$ comes from the sums $\sum_{\kappa_{1}=1}^{s} \cdots \sum_{\kappa_{h}=1}^{s}$ in (2.5). Note that $p_{\mathrm{M}, n, n, s}^{(h)}$ does not depend on $\kappa_{m}(m=1, \cdots, h)$. Plugging (2.6) to (2.7) and evaluating the sum by converting an integral leads to

$$
S_{A}=(2 \ln s) \sqrt{\frac{2 \sigma n}{\pi}}+\frac{1}{2} \ln n+\frac{1}{2} \ln (2 \pi \sigma)+\gamma-\frac{1}{2}-\ln s
$$

up to terms vanishing as $n \rightarrow \infty[3,5] . \gamma$ is the Euler constant. For $s>1$ (colored) case, the EE grows as a square root of the volume $(\sqrt{n})$, exhibiting extraordinary large entanglement. For $s=1$ (uncolored) case, the first term of (2.8) vanishes and reduces to a logarithmic growth [6].

\section{SIS Motzkin spin chain}

In this section, we consider an extension of the Motzkin spin chain by SISs. Although semigroups are generalizations of groups by relaxing the condition of the inverse, we can still introduce an inverse for every element but with respect to a partial identity. The SISs are analogs of the permutation group in group theory denoted by $\mathscr{S}_{p}^{k}$, whose element is a map defined on order- $p$ subsets of $\{1, \cdots, k\}$, expressed as $x_{a_{1} \cdots a_{p}, b_{1} \cdots b_{p}}$ with $\left\{a_{1} \cdots a_{p}\right\}$ and $\left\{b_{1} \cdots b_{p}\right\}$ being order- $p$ subsets of $\{1, \cdots, k\}$. The elements of $\mathscr{S}_{1}^{k}: x_{a, b}(a, b \in\{1, \cdots, k\})$ satisfy the product rule

$$
x_{a, b} * x_{c, d}=\delta_{b, c} x_{a, d}
$$

which is similar to that of matrices. For $p>1$, the elements of $\mathscr{S}_{p}^{k}$ can be expressed by $p$ ! sets of $\mathscr{S}_{1}^{K}$ with $K \equiv\left(\begin{array}{c}k \\ p\end{array}\right)$ whose elements are denoted by $x_{a, b}^{s}(a, b \in\{1, \cdots, K\}, s \in\{1, \cdots, p !\})$ [4]. Here we work with simplest and nontrivial cases of $\mathscr{S}_{1}^{3}$ and $\mathscr{S}_{2}^{3}$. 
For each element of $\mathscr{S}_{1}^{3}$, we introduce a spin state $\left|x_{a, b}\right\rangle$ with the canonical inner product $\left\langle x_{a, b} \mid x_{c, d}\right\rangle=\delta_{a, c} \delta_{b, d}$. Similar to the previous section, spin configurations correspond to walks in $(x, y)$-plane by regarding $\left|x_{a, b}\right\rangle$ with $a<b$ as up-steps, with $a>b$ as down-steps and with $a=b$ as flat-steps. Reflecting (3.1), a spin configuration on the sites form a connected path when spins of every adjacent site $\left|\left(x_{a, b}\right)_{j}\right\rangle\left|\left(x_{c, d}\right)_{j+1}\right\rangle(j \in\{1,2, \cdots, 2 n-1\})$ satisfy $b=c$.

The Hamiltonian for the length- $2 n$ chain is defined by

$$
H_{S 31}=H_{\text {bulk }}+H_{\text {bulk }, \text { disc }}+H_{\text {bdy }},
$$

where $H_{\text {bulk }}$ consists of four kinds of nearest-neighbor interactions corresponding to the moves in Fig. 2. $H_{\text {bulk,disc }}$ lifts disconnected paths to excited states:

$$
H_{\text {bulk,disc }}=\sum_{j=1}^{2 n-1} \sum_{a, b, c, d=1 ; b \neq c}^{3} \Pi^{\left|\left(x_{a, b}\right)_{j},\left(x_{c, d}\right)_{j+1}\right\rangle}
$$

with $\Pi^{|\psi\rangle}$ denoting the projection operator to $|\psi\rangle$. The boundary term is given by

$$
H_{\mathrm{bdy}}=\sum_{a>b} \Pi^{\left|\left(x_{a, b}\right)_{1}\right\rangle}+\sum_{a<b} \Pi^{\left.\left.\mid\left(x_{a, b}\right)\right)_{2 n}\right\rangle}+\Pi^{\left|\left(x_{1,3}\right)_{1},\left(x_{3,2}\right)_{2},\left(x_{2,1}\right)_{3}\right\rangle}+\Pi^{\left|\left(x_{1,2}\right)_{2 n-2},\left(x_{2,3}\right)_{2 n-1},\left(x_{3,1}\right)_{2 n}\right\rangle} .
$$

The last two terms in (3.4) have no analog in the original Motzkin spin chain. We can see that the Hamiltonian (3.2) is frustration free and has zero-energy ground states corresponding to connected paths starting at the origin, ending at $(2 n, 0)$ and not entering $y<0$. In this $\mathscr{S}_{1}^{3}$ version of the Motzkin walks, the maximum height in the first $n$ steps is no longer $n$ as in the original Motzkin walks but $h_{\max }=[(n-2) / 3]+2$, where $[m]$ is the greatest integer not exceeding $m$. The ground states have five-fold degeneracy according to the initial and final semigroup indices: $(1,1),(1,2)$, $(2,1),(2,2)$ and $(3,3)$ sectors. Among them, the $(3,3)$ sector is trivial and consisting of only one path $x_{3,3} x_{3,3} \cdots x_{3,3}$.
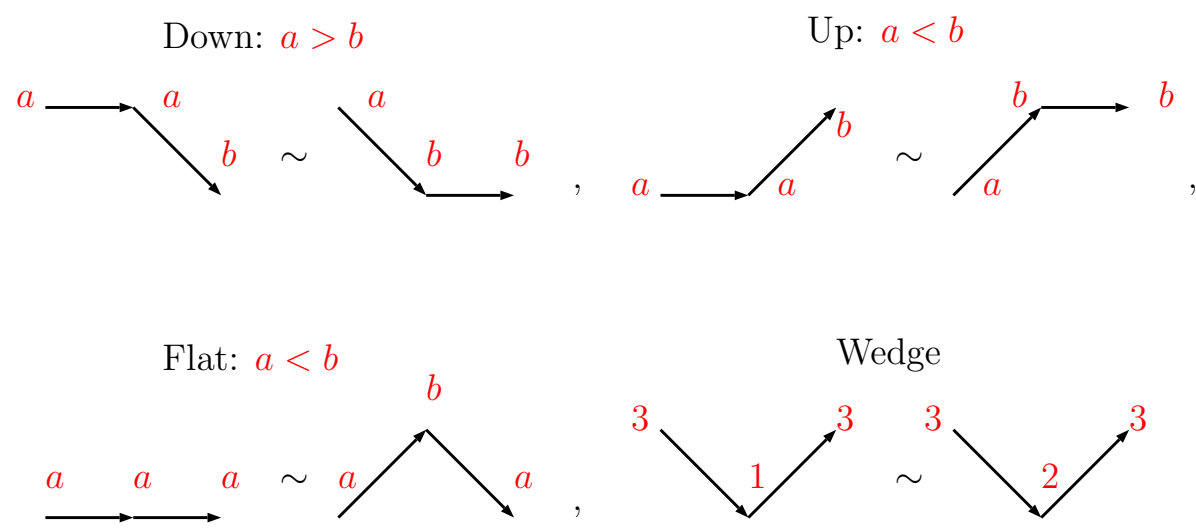

Figure 2: The moves for interaction terms in $H_{\text {bulk }}$.

After computing the numbers of the $\mathscr{S}_{1}^{3}$ Motzkin walks by using recursion relations, the EEs of a subsystem $A=\{1,2, \cdots, n\}$ can be computed as in the previous section. The EEs for the four sectors except for the $(3,3)$ are found to have the same asymptotic form as $(2.8)$ with $s=1$, i.e. violating the area law by a logarithm, whereas the $\mathrm{EE}$ for the $(3,3)$ vanishes. 
As mentioned in the above, the case of $\mathscr{S}_{2}^{3}$ can be expressed by two sets of $\mathscr{S}_{1}^{3}$, which introduces two colors to the $\mathscr{S}_{1}^{3}$ spin chain (3.2). We find that the EEs for the colored case in the sectors of $(1,1),(1,2),(2,1)$ and $(2,2)$ show the common behavior as

$$
S_{A}=(2 \ln 2) \sqrt{\frac{2 \sigma n}{\pi}}+\frac{1}{2} \ln n+\frac{1}{2} \ln (2 \pi \sigma)+\gamma-\frac{1}{2}+\ln \frac{3}{2^{1 / 3}}
$$

with $\sigma=(\sqrt{2}-1) /(9 \sqrt{2})$, up to terms vanishing as $n \rightarrow \infty[4]$. This exhibits a square-root violation of the area law similar to the original colored Motzkin model (2.8).

\section{Discussions}

We have discussed the Motzkin spin chain, which provides the extraordinary large EE growing as a square root of the volume, and its extension by SISs. For the $\mathscr{S}_{2}^{3}$ case, by adjusting a coupling constant in the Hamiltonian, the two $\mathscr{S}_{1}^{3}$ spins can be decoupled and thus the EEs grow as a logarithm of the volume. Namely, the system undergoes a quantum phase transition where the square-root growth of the EEs abruptly changes to the logarithmic growth as varying the coupling [4]. A similar spin chain with only up and down spins has been constructed by Salberger and one of the authors (V.K.) [7] (also see [8]), for which SIS extensions are discussed in [9]. As a typical feature of these SIS extensions, there are excited states corresponding to disconnected paths. Localization phenomena take place in such excited states without introducing disorders [9].

For future directions, it will be interesting to discuss the continuum limit of the spin chains and their applications to quantum gravity or black holes.

\section{References}

[1] J. Eisert, M. Cramer, M.B. Plenio, Colloquium: Area laws for the entanglement entropy, Rev. Mod. Phys. 82 (2010) 277 [arXiv: 0808 . 3773].

[2] M.B. Hastings, An area law for one-dimensional systems, J. Stat. Mech. Theory Exp. (2007) P08024 [arXiv:0705.2024].

[3] R. Movassagh, P.W. Shor, Supercritical entanglement in local systems: Counterexample to the area law for quantum matter, Proc. Natl. Acad. Sci. 113 (2016) 13278-13282 [arXiv: 1408.1657].

[4] F. Sugino, P. Padmanabhan, Area law violations and quantum phase transitions in modified Motzkin walk spin chains, J. Stat. Mech. Theory Exp. 1801 (2018) 013101 [arXiv:1710 .10426].

[5] F. Sugino, V. Korepin, Rényi entropy of highly entangled spin chains, to appear in Int. J. Mod. Phys. B [arXiv:1806.04049].

[6] S. Bravyi, L. Caha, R. Movassagh, D. Nagaj, P.W. Shor, Criticality without frustration for quantum spin-1 chains, Phys. Rev. Lett. 109 (2012) 207202 [arXiv: 1203 . 5801].

[7] O. Salberger, V. Korepin, Entangled spin chain, Rev. Math. Phys. 29 (2017) 1750031 [arXiv: 1605.03842].

[8] L. Dell'Anna, O. Salberger, L. Barbiero, A. Trombettoni, V.E. Korepin, Violation of cluster decomposition and absence of light-cones in local integer and half-integer spin chains, Phys. Rev. B 94 (2016) 155140 [arXiv: 1604 .08281].

[9] P. Padmanabhan, F. Sugino, V. Korepin, Quantum phase transitions and localization in semigroup Fredkin spin chain, arXiv:1804.00978. 